\title{
COMMENTARY
}

\section{Clinical correlates of arterial lactate levels in STEMI patients}

\author{
Max Harry Weil* and Wanchun Tang \\ See related research by Vermeulen et al., http://ccforum.com/content/14/5/R164
}

\begin{abstract}
Increases in blood lactate reflect decreases in systemic blood flows associated with low blood flow states characteristic of circulatory shock. Accordingly, the report by Vermeulen and colleagues documents the use of the blood lactate measurement as a prognostic indicator in settings of ST elevation myocardial infarction. That lactate value therefore identified highrisk patients as a complication, often with clinical signs of cardiogenic shock of corresponding severities.
\end{abstract}

The contribution by Vermeulen and associates documents measurements of blood lactate in an impressively large number of patients with ST elevation myocardial infarction [1]. On admission to the cardiac catheterization laboratory, increases in arterial blood lactate in patients corresponded to the magnitude of the infarct. Some of the patients had clinical signs of circulatory shock, including increases in heart rate and decreases in arterial pressure. If there was less successful restoration of coronary blood flow after catheter interventions, disproportionately higher blood lactate concentrations were observed.

Increases in arterial blood lactate in coronary settings were therefore most probably accompanied by decreases in cardiac output and thus by decreases in systemic blood flows consistent with cardiogenic shock of corresponding severities. To the extent that lactate measurements were obtained early after admission, they were indeed likely to be predictive of both short-term and long-term outcomes. In accordance with the historically useful Killip classification, the close relationship among the outcomes in patients undergoing primary percutaneous coronary interventions was confirmed by DeGeare and colleagues

${ }^{*}$ Correspondence: weilm@weiliccm.org

Weil Institute of Critical Care Medicine, 35100 Bob Hope Drive, Rancho Mirage, CA 92270, USA
[2]. Specifically, patients without physical signs characteristic of reduced systemic blood flows due to heart failure after acute myocardial infarction had a favorable outcome, and patients with reduction in cardiac output and heart failure had a poor prognosis.

Increases in lactate in settings of cardiogenic shock were first reported by our group in 1974 [3] and secured the earlier findings $[4,5]$ that lactate measurement is highly predictive for outcomes of circulatory shock states. In the present report, myocardial infarction led to reduced cardiac work capacity and therefore reduced systemic blood flows. The increases in lactate were then best explained by critical reduction in cardiac output, which is typically accompanied by physical signs, including tachycardia hypotension, cyanosis and pallor, third heart sounds and cold extremities. The sources of the lactate increase may in part be due to ischemic myocardium, but the likelihood is that lactic excesses were primarily due to systemic circulatory failure. Unrelated causes of increased lactate are associated with struggling, convulsive seizures and hyperthermia, and must be excluded [6].

The evolution of lactate measurement followed the early studies of Huckabee $[7,8]$ who, largely on the basis of earlier investigations in exercise physiology and based on measurements of both serum pyruvate and lactate values, clarified the value of the so-called excess lactate as a quantitator corresponding to the systemic oxygen deficit [4]. Subsequent workers confirmed that excess lactate was a valuable measurement and yet later demonstrated that the measurement of lactate alone in blood or serum was sufficient [5,8-10]. With present methods of facile point-of-care laboratory measurements, as utilized in the study by Vermeulen and colleagues [1], lactate proved a useful prognosticator for the severity of myocardial infarction. When acute myocardial infarction was accompanied by lactic acidosis, systemic blood flow was critically reduced.

The present report, therefore, again demonstrates the early prognostic value of lactate as an indicator for the severity of decreased systemic blood flows with correspondingly poor outcomes and, in this instance, in 
catheter laboratory settings of ST elevation acute myocardial infarction.

\section{Abbreviations}

STEMI, ST elevation myocardial infarction.

\section{Competing interests}

The authors declare that they have no competing interests.

\section{Acknowledgements}

The present work was supported by The Weil Family Foundation.

Published: 26 January 2011

\section{References}

1. Vermeulen RP, Hoekstra M, vd Horst ICC, van Pelt LJ, Jessurun GAJ, Jaarsma T, van den Heuvel AFM, Zilstra F: Clinical correlates of arterial lactate levels in patients with ST-segment elevation myocardial infarction at admission: a descriptive study. Crit Care 2010, 14:R164.

2. DeGeare VS, Boura JA, Grinnes LL, O'Neill WW, Grines CL: Predictive value of the Killip classification in patients undergoing primary percutaneous coronary intervention for acute myocardial infarction. Am J Cardio/ 2001, 87:1035-1038

3. Afifi AA, Chang PC, Liu VY, Da Luz P, Weil MH, Shubin H: Prognostic indexes in acute myocardial infarction complicated by shock. Am J Cardiol 1974, 33:826-832.
4. Broder $\mathrm{G}$, Weil MH: Excess lactate: an index of reversibility of shock in human patients. Science 1964, 143:1457-1459.

5. Weil MH, Afifi AA: Experimental and clinical studies on lactate and pyruvate as indicators of the severity of acute circulatory failure (shock). Circulation 1970, 41:989-1001.

6. Orringer CE, Eustace JC, Wunch CD, Gardner LB: Natural history of lactic acidosis after grand-mal seizures. A model for the study of an anion-gap acidosis not associated with hyperkalemia. N Engl J Med 1977, 297:796-799.

7. Huckabee WE: Abnormal resting blood lactate: I. The significance of hyperlactatemia in hospitalized patients. Am J Med 1961, 30:833-839.

8. Huckabee WE: Abnormal resting blood lactate: II. Lactic acidosis. Am J Med 1961, 30:840-848.

9. Vincent JL, Dufaye P, Berré J, Lerman M, DeGaute JP, Kahn RJ: Serial lactate determinations during circulatory shock. Crit Care Med 1983, 11:449-451.

10. Mizock BA, Falk JL: Lactic acidosis in critical illness. Crit Care Med 1992, 20:80-93.

doi:10.1186/cc9383

Cite this article as: Weil MH, Tang W: Clinical correlates of arterial lactate levels in STEMI patients. Critical Care 2011, 15:113. 